\section{Designing Materials for Extreme Environments}

Meeting the world's demand for energy depends on the existence of materials that can function in extreme environments, such as the interior of a nuclear reactor or deep underwater. Developing these materials requires increased investments in many areas of basic materials research, according to participants in a recent workshop sponsored by the Department of Energy's Office of Basic Energy Sciences (BES).

"When things fail, they often do so because materials fail," said Jeff Wadsworth of Oak Ridge National Laboratory, chair of the workshop. "The environment we place materials in has a huge influence on how and when things fail...so to the extent our knowledge is limited, so do we place limitations on our materials."

Nearly 100 panelists from around the world and across the spectrum of laboratories and research centers converged on a set of current knowledge limitations during the course of the workshop. Overcoming these limitations, they believe, needs to be high priority for researchers and funding agencies.

Four environments were consideredenergetic flux extremes, chemical extremes, thermomechanical extremes, and electromagnetic extremes.

Within each of these extreme environments, panelists considered the following:

- how materials behave under extreme conditions;

- how extreme environments can assist in the creation of new materials that address current needs; and

- how to make revolutionary rather than evolutionary advances.

Participants in the panel on energetic flux extremes recommended that high

\section{ESF Issues Call for Research Networking Programs Proposals}

The European Science Foundation (ESF) Research Networking Programs consist of networking activities bringing together nationally funded research activities for four to five years to address major scientific issues or science-driven topics of research infrastructure at the European level with the aim of advancing the frontiers of science. More information is available at Web site www. esf.org/activities/research-networking-programmes / 2007-call-forproposals.html/. The submission deadline is October 30, 2007. priority be placed on basic research aimed at equipping materials with revolutionary tolerance to extreme photon and particle fluxes. In addition, they cited as high priority the ability to use energetic fluxes to synthesize new materials far from equilibrium.

As the demand for power increases, panelists said, so does the demand for materials that can function in energetic flux extremes, such as inside nuclear reactors. These harsh environments degrade materials and can significantly reduce the lifetimes of sensors and control systems.

The chemical extremes panel called for increased knowledge and control of surfaces at the atomic scale, which could enable the creation of non-reacting interfaces. In addition, they called for the establishment of guiding principles for materials in conditions where chemical reaction dynamics leads to new paradigms. Technological advances in fuel cells, steam plants, turbines, and many other areas are limited by the materials' ability to withstand chemical extremes.

The universe has an enormous range of temperatures and pressures, and priority research directions for materials in such thermomechanical extremes include studying chemical and materials dynamics in complex systems in real time and exploring disordered materials, according to the panel.

In particular, the panel emphasized the value of being able to predict the response of new materials based on computational simulation. This would enable the functional design of disordered materials whose performances are difficult to measure.

Priority research areas for materials in electromagnetic extremes include designing materials with higher mechanical strength, higher dielectric strength, and lower electrical conductivity than currently available. In addition, the panelists said, researchers need to understand dielectric breakdown at a fundamental level and identify the physical mechanisms that govern the fundamental limits of performance.

A separate group of panelists was charged with exploring the priorities that cut across all four extremes. They advocated creating a comprehensive theoretical and simulation framework for understanding materials in extreme environments.

In addition, the cross-cutting panel recommended high priority be placed on experiments that probe materials on the scale of fundamental interactions, designing and synthesizing materials that reach their fundamental performance limits, and exploiting extreme environments as a control for materials design and synthesis. The grand challenge they put forth is to achieve the fundamental failure limit of materials as defined by their intrinsic, theoretical performance capacities.

Wadsworth said that the time has come for revolutionary advances in materials research for two reasons: the current energy demands and the convergence of theory and experiments.

Robert Romanosky of the Department of Energy fossil energy program put the energy challenge bluntly during one of the plenary sessions when he told participants, "We're going to need every piece of power that we can generate." He continued, "Everything is going to play a piece but they all come down to one common factor, they all need materials research."

In addition to this motivating need, Wadsworth said, revolutionary advances in materials research are now possible because theory and experiment are converging at a level that allows researchers to explore the range of extreme conditions more fully than before.

A report detailing the workshop results will be available to the public on the BES Web site, www.er.doe.gov/bes/BES.html, and will be distributed to policy makers. Director of the Office of Basic Energy Sciences, Patricia Dehmer, said that, like previous BES reports, she expects this report will have great impact on the community and policy makers alike.

KENDRA RAND

\section{Nanotechnology in New Zealand: Small Research with a Big Impact?}

New Zealand is a small country of 4.1 million people in the South Pacific, and by many measures it is a long way from anywhere. The flight from Los Angeles or Singapore takes 10 hours, and even Sydney is 3 hours away. New Zealand's capital city, Wellington, is the southernmost national capital in the world, and the city of Christchurch serves as the primary staging base for many international Antarctic research missions.

New Zealand's economy has traditionally been mainly agricultural, exporting large quantities of dairy products, meat, and lumber to Australia, the United States, the United Kingdom, and Asia. For the past 20 years, the New Zealand government has worked to move its economy toward a more industrialized, globally competitive base, with some success. As part of this effort, the New Zealand Ministry of Research, Science \& Technology (MoRST) has released a series of Roadmaps for Science, to guide science and technology efforts in various fields. In December 2006 the Roadmap 
for Nanoscience and Nanotechnologies was released, with a significant materials science component.

Public investment in nanoscience and nanotechnologies in New Zealand is currently USD\$8 million, which is approximately the same fraction of the gross domestic product $(0.1 \%)$ as the National Nanotechnology Initiative in the United States. Most of this nanoscience and nanotechnology work is focused on basic research, and New Zealand has developed significant research strengths in nanoelectronics, optoelectronics, and advanced composite materials. Much of this work is conducted at the MacDiarmid Institute for Advanced Materials and Nanotechnology (based at Victoria University of Wellington and the University of Canterbury) and the University of Auckland, as well as several Crown Research Institutes. Nano-related research at New Zealand universities has generated a small number of spin-off companies, most notably Nano Cluster Devices Ltd., which offers self-assembling nanowires based on atomic cluster deposition.

The Roadmap lays out a short-term policy of continuing to emphasize basic nanorelated research until 2010. In the medium term (to 2015) it establishes a policy of emphasizing nano-related research that is aimed at developing technology relevant to New Zealand's existing industries rather than seeking revolutionary new applications of nanoscience. These existing industries are mostly in biologically oriented fields such as agriculture, forestry, and food packaging, which the Roadmap specifically notes should be a focus of this research. In fact, some bio-nanotechnology research is already being conducted in New Zealand, including work at Scion/ Forest Research Institute Ltd. to develop organic nanocomposite materials based on wood cellulose that may eventually lead to biodegradable, high-strength engineering materials and textiles. The Roadmap notes that nanoscience and nanotechnology work that contributes to productivity improvements and environmental sustainability in existing industries is likely to have the greatest national benefit in the short and medium term. This kind of research outcome is also most likely to result from increased collaboration both within New Zealand and internationally, which the Roadmap urges. The Roadmap notes that in the long term, revolutionary benefits to New Zealand from nanoscience are possible, but beyond a general commitment to scientific excellence it does not specify a strategy for achieving them.

The other key policy guidance set by the Roadmap is the need for social science research to inform the direction of nano-related research in New Zealand, and the importance of public engagement in nano-related policy decisions. For both economic and social reasons, New Zealand places a high value on environmental sustainability, which is important for the agricultural sector and the growing tourism industry (tourism surpassed dairy products as New Zealand's largest foreign revenue source in 2003) and future nanoscience and nanotechnology policy will continue to take this into account. Also, the Roadmap states that New Zealand should actively participate in international standards-setting for characterizing nanoparticles and ensuring their safety, particularly in food preparation, packaging, and processing. The MoRST expects this Roadmap to remain in effect for 5 to 10 years, although it will revisit it periodically and formally consider an update by 2011.

COLIN MCCORMICK

\section{India's Bio-Fuel Program to be Developed in Public-Private Partnership}

The Bio-Fuel Program in India is to be developed in Public-Private Partnerships which would promote bio-fuel plantations on government, community, and private lands. Simultaneously, the industrial sector is being encouraged to increase ethanol production from non-food feedstocks. Addressing the International Bio-Fuels Conference held in Brussels last summer, Vilas Muttemvar, the Minister for New and Renewable Energy, said that several Indian corporates and business houses have in collaboration with the government started to establish Jatropha plantations on waste lands or have begun contract farming on private holdings. Oil is extracted from the seeds of the Jatropha plant and is mixed with diesel to form bio-fuels.

Muttemvar said that several state governments have announced policies to encourage Jatropha cultivation, setting up bio-diesel plants and supply chains in many states.

The Minister said that the national government is in the process of preparing a national policy on bio-fuels that is expect- ed to lay the foundation for the accelerated development of the sector. The areas likely to be covered in the policy include research and development. The policy will aim at a short-term blending proportion of $5 \%$ by 2012 , medium term of $10 \%$ by 2017, and a long-term of above beyond 2017 and the policy would also recommend fixation of minimum support price for Jatropha and other nonedible oilseeds.

The Minister said that the focus of India's bio-fuel program is on indigenous development to the extent possible by using science and technology and industrial capabilities. The Minister expects India to work with the European Union and all participating countries for achieving India's bio-fuel aims and targets. India's priorities for cooperation include programs for increasing crop yields, oil content of oilseeds, and reducing the environmental impact of bio-fuel usage. The government is also looking at cooperation in fuel conversion technologies from waste cellulosic materials and dedicated cellulosic crops.

The Minister further emphasized that bio-fuels are an integral component of India's National Energy Policy.

Over these years, contribution of renewable energy has increased significantly in India, meeting about $15 \%$ of the country's electricity needs, of which $12 \%$ comes from large hydroelectric generators. Around 45,000 MW renewable power generating capacity to date or one-third of the total has been installed comprising over $7000 \mathrm{MW}$ from wind, $1200 \mathrm{MW}$ from bio power, and over 36,000 MW from hydro power of which $2000 \mathrm{MW}$ is from small hydro power. Muttemvar further said that India has met with success in solar hot water systems area. Two million square meters of collector area has been installed so far and the government is planning to add an additional 10 million square meters during the next five years.

Muttemvar said that a National Hydrogen Energy Roadmap for the introduction of a hydrogen economy has been formulated and the preparation of the Roadmap entailed detailed discussions among policy makers, scientists, and corporations. Muttemvar said that India is aiming to add one million hydrogen-powered two-, three-, and four-wheelers by 2020.

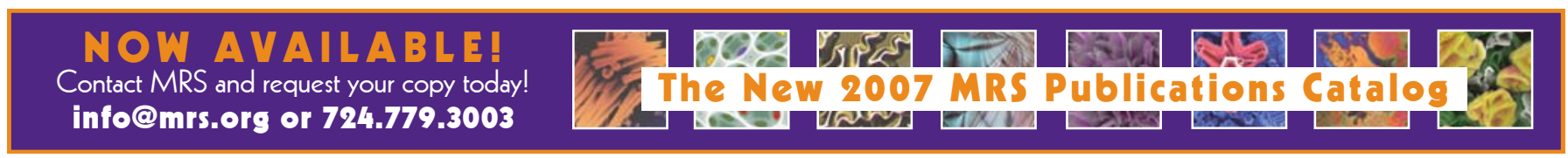

